\title{
LOCALIZABLE SPECTRUM AND BOUNDED LOCAL RESOLVENT FUNCTIONS
}

\author{
VLADIMIR MÜLLER AND MICHAEL M. NEUMANN
}

\begin{abstract}
Given a Banach space operator with interior points in the localizable spectrum and without non-trivial divisible subspaces, this article centers around the construction of an infinite-dimensional linear subspace of vectors at which the local resolvent function of the operator is bounded and even admits a continuous extension to the closure of its natural domain. As a consequence, it is shown that, for any measure with natural spectrum on a locally compact abelian group, the corresponding operator of convolution on the group algebra admits a non-zero bounded local resolvent function precisely when its spectrum has non-empty interior.

Mathematics Subject Classification (2000). Primary 47A11; Secondary 43A25, 47A10, 47B40.
\end{abstract}

\section{INTRODUCTION}

González [5] was the first to observe that, in remarkable contrast to the case of the usual resolvent function, bounded linear operators on Banach spaces may well admit non-trivial bounded local resolvent functions. In fact, as shown in Theorem 2 of [2], a normal operator admits a non-zero bounded local resolvent function precisely when its spectrum has non-empty interior. Moreover, by Proposition 2.1 of [13], this characterization remains valid for all multiplication operators on the space of continuous functions on a compact Hausdorff space; see also Sections 1.2 and 1.5 of [10]. One might expect a similar result to hold for convolution operators on group algebras, since such operators are, via the Fourier-Stieltjes transform, intimately related to multiplication operators. The question of the existence of non-zero bounded local resolvent functions for convolution operators was stated as an open problem in [13], [14], and [10, 6.2.14].

In this article, we provide a positive solution to this problem for the case of convolution by measures that avoid the Wiener-Pitt phenomenon. In fact, in Section 5 , we settle the more general case of multipliers with natural spectrum on a regular semi-simple commutative Banach algebra. This result is a consequence of a new method of constructing bounded local resolvent functions for a general class of operators in Section 4. Under mild conditions on a bounded linear operator $T$ on a Banach space $X$, we find an infinite-dimensional subspace $Y$ of $X$ such that, for each $y \in Y$, the local resolvent function of $T$ at $y$ is not only bounded on its natural domain, the local resolvent set $\rho_{T}(y)$, but even has a continuous extension to the closure of $\rho_{T}(y)$. Our approach is based on a certain closed subset of the usual spectrum, the localizable spectrum $\sigma_{\text {loc }}(T)$, which happens to coincide with the spectrum in the case of decomposable operators and of multipliers with natural 
spectrum. We also need a certain algebraic version of the classical single-valued extension property. This condition and some other preliminaries from local spectral theory are discussed in Sections 2 and 3.

\section{Algebraic CORE And Algebraic spectral subspaces}

Throughout this section, let $T$ be a linear operator acting on an arbitrary complex vector space $X$. Following Saphar [15], the algebraic core $C(T)$ of $T$ is defined to be the linear span of all linear subspaces $M$ of $X$ for which $T M=M$. Thus $C(T)$ is simply the largest subspace $M$ with this property. The elementary proof of the following result may be found in Theorem 1.8 of [1].

Lemma 1. $C(T)$ consists precisely of all vectors $x_{0} \in X$ for which there exist $x_{n} \in X$ such that $T x_{n}=x_{n-1}$ for all $n \in \mathbb{N}$.

Similarly, for an arbitrary subset $F$ of $\mathbb{C}$, let $E_{T}(F)$ denote the largest linear subspace $M$ of $X$ for which $(T-\lambda) M=M$ for all $\lambda \in \mathbb{C} \backslash F$. The spaces $E_{T}(F)$ are known as the algebraic spectral subspaces of $T$. They were first introduced, via transfinite induction, by Johnson and Sinclair [7] in the context of automatic continuity theory, but then proved to be a useful tool in local spectral theory as well; see Sections 1.4, 1.5, 5.4, and 5.5 of [10] and also 2.4 of [1]. Of particular interest is the space $E_{T}(\emptyset)$, often called the largest divisible subspace for $T$. In the theory of automatic continuity of intertwining linear transformations, it is essential to avoid non-trivial divisible subspaces. In the same vein, the condition $E_{T}(\emptyset)=\{0\}$ will be required in the main results of the present article.

Clearly, $E_{T}(\mathbb{C} \backslash\{\lambda\})=C(T-\lambda)$ for each $\lambda \in \mathbb{C}$. This identity will be extended in Proposition 3 below, based on the following simple result.

Lemma 2. Let $A, B, C, D$ be mutually commuting linear operators on $X$, and suppose that $A C+B D=I$, the identity operator on $X$. Let $u, v \in X$ satisfy $A u=B v$. Then there exists some $y \in X$ such that $B y=u$ and $A y=v$.

Proof. Set $y:=D u+C v$. Then $B y=B D u+B C v=(I-A C) u+C A u=u$ and, similarly, $A y=A D u+A C v=D B v+(I-B D) v=v$, as desired.

Proposition 3. $E_{T}(F)=\bigcap_{\lambda \in \mathbb{C} \backslash F} C(T-\lambda)$ for each subset $F$ of $\mathbb{C}$.

Proof. Given $F \subseteq \mathbb{C}$, let $M:=\bigcap_{\lambda \in \mathbb{C} \backslash F} C(T-\lambda)$. Clearly, $E_{T}(F) \subseteq C(T-\lambda)$ for each $\lambda \in \mathbb{C} \backslash F$, and hence $E_{T}(F) \subseteq M$. To establish the converse inclusion, we have to show that $(T-\lambda) M=M$ for each $\lambda \in \mathbb{C} \backslash F$. Since $M$ is certainly invariant under $T$, it suffices to find, for arbitrary $\lambda \in \mathbb{C} \backslash F$ and $x_{0} \in M$, some vector $y_{0} \in M$ such that $(T-\lambda) y_{0}=x_{0}$.

Because $x_{0} \in M \subseteq C(T-\lambda)$, there exists some $y_{0} \in C(T-\lambda)$ for which $(T-\lambda) y_{0}=x_{0}$. We claim that actually $y_{0} \in M$, i.e., $y_{0} \in C(T-\mu)$ for each $\mu \in \mathbb{C} \backslash F$. This is clear for $\mu=\lambda$, so we may assume that $\mu \neq \lambda$.

Since $x_{0} \in M \subseteq C(T-\mu)$, Lemma 1 leads to a sequence of vectors $x_{n} \in X$ with $(T-\mu) x_{n}=x_{n-1}$ for all $n \in \mathbb{N}$. Note that $(T-\mu) I /(\lambda-\mu)+(T-\lambda) I /(\mu-\lambda)=I$. Because $(T-\mu) x_{1}=x_{0}=(T-\lambda) y_{0}$, there exists, by Lemma 2 , some $y_{1} \in X$ such that $(T-\lambda) y_{1}=x_{1}$ and $(T-\mu) y_{1}=y_{0}$. Now $(T-\mu) x_{2}=x_{1}=(T-\lambda) y_{1}$, so, again by Lemma 2 , there exists some $y_{2} \in X$ such that $(T-\lambda) y_{2}=x_{2}$ and $(T-\mu) y_{2}=y_{1}$. Inductively, we may construct vectors $y_{n} \in X$ such that $(T-\lambda) y_{n}=x_{n}$ and $(T-\mu) y_{n}=y_{n-1}$ for all $n \in \mathbb{N}$. Thus, again by Lemma $1, y_{0} \in C(T-\mu)$ for all 
$\mu \in \mathbb{C} \backslash F$ and therefore $x_{0}=(T-\lambda) y_{0} \in(T-\lambda) M$. This completes the proof of the inclusion $M \subseteq E_{T}(F)$.

Proposition 3 may also be deduced from the fact that the algebraic spectral subspaces preserve arbitrary intersections, in the sense that

$$
E_{T}\left(\bigcap_{\alpha \in A} F_{\alpha}\right)=\bigcap_{\alpha \in A} E_{T}\left(F_{\alpha}\right)
$$

for any family $\left(F_{\alpha}\right)_{\alpha \in A}$ of subsets of $\mathbb{C}$. This result was established by Laursen [9] based on different methods; see also Theorem 2.69 of [1]. We note that, conversely, Proposition 3 leads to a considerably shorter new proof of Laursen's result.

\section{TOOLS FROM LOCAL SPECTRAL THEORY}

We now review a few notions from local spectral theory, with emphasis on the analytic counterparts of the algebraic core and the algebraic spectral subspaces; see [1] and [10].

Given a bounded linear operator $T \in L(X)$ on a complex Banach space $X$, let $\rho(T)$ and $\sigma(T)$ denote, as usual, the resolvent set and the spectrum of $T$. The analytic core $K(T)$ of $T$ is defined as the space consisting of all $x_{0} \in X$ for which there exists a constant $c>0$ and a sequence of vectors $x_{n} \in X$ such that $T x_{n}=$ $x_{n-1}$ and $\left\|x_{n}\right\| \leq c^{n}$ for all $n \in \mathbb{N}$. Clearly, by Lemma $1, K(T) \subseteq C(T)$, and, by Theorem 1.22 of [1], equality occurs whenever $C(T)$ is closed. In particular, by Theorems 1.10 and 1.24 of [1], the identities $K(T)=C(T)=\bigcap_{n=1}^{\infty} T^{n} X$ hold provided that $T$ is semi-regular or a semi-Fredholm operator.

The local resolvent set $\rho_{T}(x)$ of $T$ at a vector $x \in X$ is defined as the set of all $\lambda \in \mathbb{C}$ for which there exist an open neighborhood $U$ of $\lambda$ in $\mathbb{C}$ and an analytic function $f: U \rightarrow X$ such that $(T-\mu) f(\mu)=x$ for all $\mu \in U$. The local spectrum of $T$ at $x$ is the set $\sigma_{T}(x):=\mathbb{C} \backslash \rho_{T}(x)$. We will use the elementary fact that $\sigma_{T}(u+v) \subseteq \sigma_{T}(u) \cup \sigma_{T}(v)$ for arbitrary $u, v \in X$ and that equality holds in this inclusion provided that $\sigma_{T}(u) \cap \sigma_{T}(v)=\emptyset$.

Also, for each $F \subseteq \mathbb{C}$, let $X_{T}(F)$ consist of all $x \in X$ for which $\sigma_{T}(x) \subseteq F$. The spaces $X_{T}(F)$ are called the local spectral subspaces of $T$. Since, by Proposition 1.2.16 of [10], $(T-\lambda) X_{T}(F)=X_{T}(F)$ for all $\lambda \in \mathbb{C} \backslash F$, we have $X_{T}(F) \subseteq E_{T}(F)$ for each $F \subseteq \mathbb{C}$. Moreover, by Proposition 1.4.14 of [10], equality holds for each closed subset $F$ of $\mathbb{C}$ provided that $T$ is super-decomposable with $E_{T}(\emptyset)=\{0\}$.

By Proposition 3.3.7 of [10], $X_{T}(\mathbb{C} \backslash\{\lambda\})=K(T-\lambda)$ for all $\lambda \in \mathbb{C}$. Since the local spectral subspaces clearly preserve arbitrary intersections, we thus obtain the analytic counterpart of Proposition 3, namely the identity $X_{T}(F)=\bigcap_{\lambda \in \mathbb{C} \backslash F} K(T-$ $\lambda$ ) for each subset $F$ of $\mathbb{C}$.

The operator $T$ is said to have the single-valued extension property (SVEP) if, for each open subset $U$ of $\mathbb{C}$, the only analytic function $f: U \rightarrow X$ for which $(T-\lambda) f(\lambda)=0$ for all $\lambda \in U$ is the zero function $f \equiv 0$ on $U$. This condition dates back to Dunford and is shared by all normal, spectral, and decomposable operators as well as by all multipliers on semi-simple commutative Banach algebras; see [1] and $[10]$.

Evidently, if $T$ has (SVEP), then, for each $x \in X$, there exists a unique $X$-valued analytic function $f$ on the entire local resolvent set $\rho_{T}(x)$ for which $(T-\lambda) f(\lambda)=x$ for all $\lambda \in \rho_{T}(x)$. Let $R_{T}(\lambda, x):=f(\lambda)$ for all $\lambda \in \rho_{T}(x)$. The function $R_{T}(\cdot, x)$ is called the local resolvent function of $T$ at $x$. Clearly, $\rho(T) \subseteq \rho_{T}(x)$ and $R_{T}(\lambda, x)=$ 
$(T-\lambda)^{-1} x$ for all $\lambda \in \rho(T)$. In particular, it follows that $R_{T}(\lambda, x) \rightarrow 0$ as $|\lambda| \rightarrow \infty$. Consequently, if $R_{T}(\cdot, x)$ admits a continuous extension to the closure of $\rho_{T}(y)$, then $R_{T}(\cdot, x)$ is certainly bounded on $\rho_{T}(x)$.

\section{MAin Results}

In Theorem 4 below, the existence of bounded local resolvent functions will be established under two conditions on the operator $T \in L(X)$ on an arbitrary complex Banach space $X$.

The first requirement is that $E_{T}(\emptyset)=\{0\}$. This condition entails (SVEP) for $T$, since $X_{T}(\emptyset) \subseteq E_{T}(\emptyset)$ and, by Proposition 1.2.16 of [10], $T$ has (SVEP) precisely when $X_{T}(\emptyset)=\{0\}$. On the other hand, since $E_{T}(\emptyset) \subseteq \bigcap_{p \in \mathbb{N}} \bigcap_{\lambda \in \mathbb{C}}(T-\lambda)^{p} X$, it follows that $E_{T}(\emptyset)$ is trivial whenever the operator $T$ is normal, hyponormal, generalized scalar, or the restriction of one of such operators to a closed invariant subspace; see Section 1.5 of [10] for details. However, the classical Volterra operator $T$ on the Banach space $C([0,1])$ satisfies $E_{T}(\emptyset) \neq\{0\}$, although, as a quasi-nilpotent operator, $T$ certainly has (SVEP); see Example 1.4 .12 of [10].

The second condition involves the localizable spectrum $\sigma_{\mathrm{loc}}(T)$ of an operator $T$ with (SVEP), defined as the set of all $\lambda \in \mathbb{C}$ for which $X_{T}(V) \neq\{0\}$ for each open neighborhood $V$ of $\lambda$. As shown by Eschmeier and Prunaru [4], the localizable spectrum plays an important role in the theory of invariant subspaces; see also [3] and [11]. It is known that $\sigma_{\text {loc }}(T)$ is a closed subset of $\sigma(T)$ and that $\sigma_{\text {loc }}(T)$ contains the point spectrum and is included in the approximate point spectrum of $T$; see Theorem 2 of [11]. Moreover, by the same result, $\sigma_{\text {loc }}(T)=\sigma(T)$ when $T$ is decomposable or, more generally, the quotient of a decomposable operator by a closed invariant subspace, while $\sigma_{\text {loc }}(T)$ may well be empty when $T$ is the restriction of a decomposable operator. For instance, $\sigma_{\mathrm{loc}}(T)=\emptyset$ if $T$ is the unilateral right shift on $\ell^{p}(\mathbb{N})$ for $1 \leq p<\infty$ or any pure isometry on a Hilbert space; see Example 3 of $[11]$.

Let $D(\lambda, r)$ denote the open disc in $\mathbb{C}$ with center $\lambda \in \mathbb{C}$ and radius $r>0$.

Theorem 4. Let $T \in L(X)$ be an operator for which $E_{T}(\emptyset)=\{0\}$ and $\sigma_{\mathrm{loc}}(T)$ has non-empty interior, suppose that $U$ is a non-empty open subset of $\sigma_{\mathrm{loc}}(T)$, let $x \in X$ be a vector with $\sigma_{T}(x) \subseteq U$, and let $\varepsilon>0$. Then there exists some $y \in X$ for which $\|y-x\| \leq \varepsilon, \sigma_{T}(y)=\bar{U}$, and $R_{T}(\cdot, y)$ can be continuously extended to $\mathbb{C} \backslash U$. In particular, $T$ has a bounded local resolvent function at $y$.

Proof. Let $\left(\lambda_{n}\right)_{n \in \mathbb{N}}$ be a sequence of distinct elements of $U$ that is dense in $\bar{U}$. For each $n \in \mathbb{N}$, we then construct inductively a vector $x_{n} \in X$ with $\left\|x_{n}\right\|=1$, a complex number $\mu_{n} \in U \cap D\left(\lambda_{n}, n^{-1}\right)$, vectors $u_{n, i, j} \in X$ for all $i, j \in \mathbb{N}$ with $i<n$, and finally a real number $\alpha_{n} \geq 0$ such that the following seven conditions are fulfilled: 


$$
\begin{aligned}
& \sigma_{T}\left(x_{n}\right) \subseteq U \cap D\left(\lambda_{n}, n^{-1}\right) \backslash\left\{\mu_{1}, \ldots, \mu_{n-1}\right\} ; \\
& \left(T-\mu_{i}\right) u_{n, i, 1}=x_{n} \quad \text { for all } i \in \mathbb{N} \text { with } i<n ; \\
& \left(T-\mu_{i}\right) u_{n, i, j}=u_{n, i, j-1} \quad \text { for all } i, j \in \mathbb{N} \text { with } i<n \text { and } j \geq 2 ; \\
& x+\sum_{i=1}^{n} \alpha_{i} x_{i} \notin C\left(T-\mu_{n}\right) ; \\
& \alpha_{n} \leq 2^{-n} \varepsilon ; \\
& \sup \left\{\left\|R_{T}\left(\zeta, \alpha_{n} x_{n}\right)\right\|: \zeta \in \mathbb{C} \backslash U\right\} \leq 2^{-n} ; \\
& \alpha_{n}\left\|u_{n, i, j}\right\| \leq 2^{-n} \quad \text { for all } i, j \in \mathbb{N} \text { with } i<n \text { and } j \leq n .
\end{aligned}
$$

Let $n \in \mathbb{N}$ be given, and suppose that $x_{m}, \mu_{m}, u_{m, i, j}, \alpha_{m}$ satisfying $(1)-(7)$ have already been constructed for $m=1, \ldots, n-1$. Since $U$ is an open subset of $\sigma_{\text {loc }}(T)$, we find some $x_{n} \in X$ with $\left\|x_{n}\right\|=1$ such that condition (1) is fulfilled. Because $T$ has no non-trivial divisible subspace, we know from Proposition 3 that the intersection of the algebraic cores $C(T-\mu)$ as $\mu$ ranges through $\mathbb{C}$ is $\{0\}$. Hence there exists some $\mu_{n} \in \mathbb{C}$ such that $x_{n} \notin C\left(T-\mu_{n}\right)$. Because

$$
X_{T}\left(\mathbb{C} \backslash\left\{\mu_{n}\right\}\right)=K\left(T-\mu_{n}\right) \subseteq C\left(T-\mu_{n}\right),
$$

this ensures that $\mu_{n} \in \sigma_{T}\left(x_{n}\right)$ and therefore $\mu_{n} \in U \cap D\left(\lambda_{n}, n^{-1}\right) \backslash\left\{\mu_{1}, \ldots, \mu_{n-1}\right\}$, by the choice of $x_{n}$ satisfying condition (1). On the other hand, for each $i \in$ $\{1, \ldots, n-1\}$, we have $\mu_{i} \notin \sigma_{T}\left(x_{n}\right)$ and therefore $x_{n} \in C\left(T-\mu_{i}\right)$. Hence, by the characterization of the algebraic core from Lemma 1 , we can find vectors $u_{n, i, j} \in X$ for all $j \in \mathbb{N}$ such that conditions (2) and (3) are fulfilled.

Finally, for the construction of $\alpha_{n}$, we distinguish two cases for the vector $z:=x+$ $\sum_{i=1}^{n-1} \alpha_{i} x_{i}$. If $z \notin C\left(T-\mu_{n}\right)$, then we set $\alpha_{n}=0$, so that all the conditions $(4)-(7)$ are trivially satisfied. If, however, $z \in C\left(T-\mu_{n}\right)$, then we choose any strictly positive number $\alpha_{n}$ that is small enough so that conditions $(5)-(7)$ hold. This is possible, since the inclusion $\sigma_{T}\left(x_{n}\right) \subseteq U$ guarantees that $R_{T}\left(\cdot, x_{n}\right)$ is bounded on $\mathbb{C} \backslash U$. Because $\alpha_{n}>0, x_{n} \notin C\left(T-\mu_{n}\right)$, and $z \in C\left(T-\mu_{n}\right)$, we see that also condition (4) is satisfied. This completes our inductive construction.

Evidently, by condition (5), the series $y:=x+\sum_{i=1}^{\infty} \alpha_{i} x_{i}$ converges in $X$ and satisfies $\|y-x\| \leq \sum_{i=1}^{\infty} \alpha_{i} \leq \varepsilon$. Similarly, by condition (6), the series

$$
f(\zeta):=R_{T}(\zeta, x)+\sum_{i=1}^{\infty} R_{T}\left(\zeta, \alpha_{i} x_{i}\right)
$$

converges for each $\zeta \in \mathbb{C} \backslash U$. As the uniform limit of continuous functions, $f$ is certainly continuous on $\mathbb{C} \backslash U$. Moreover, $f$ is analytic on $\mathbb{C} \backslash \bar{U}$ and satisfies $(T-\zeta) f(\zeta)=y$ for all $\zeta \in \mathbb{C} \backslash \bar{U}$. Thus $\mathbb{C} \backslash \bar{U} \subseteq \rho_{T}(y)$ and $R_{T}(\zeta, y)=f(\zeta)$ for all $\zeta \in \mathbb{C} \backslash \bar{U}$. In particular, we have $\sigma_{T}(y) \subseteq \bar{U}$.

It remains to show that $\bar{U} \subseteq \sigma_{T}(y)$. Since, by construction, $\left|\lambda_{n}-\mu_{n}\right|<n^{-1}$ for all $n \in \mathbb{N}$ and $\left(\lambda_{n}\right)_{n \in \mathbb{N}}$ is dense in $\bar{U}$, so is $\left(\mu_{n}\right)_{n \in \mathbb{N}}$. Consequently, it is sufficient to prove that $\mu_{n} \in \sigma_{T}(y)$ for all $n \in \mathbb{N}$.

Given an arbitrary $n \in \mathbb{N}$, we observe that, for each $j \in \mathbb{N}$, condition (7) ensures that

$$
\sum_{m \geq \max \{n+1, j\}} \alpha_{m}\left\|u_{m, n, j}\right\| \leq \sum_{m \geq \max \{n+1, j\}} 2^{-m}<\infty .
$$

Hence we may define $v_{j}:=\sum_{m=n+1}^{\infty} \alpha_{m} u_{m, n, j}$. By condition (2), we have

$$
\left(T-\mu_{n}\right) v_{1}=\sum_{m=n+1}^{\infty} \alpha_{m}\left(T-\mu_{n}\right) u_{m, n, 1}=\sum_{m=n+1}^{\infty} \alpha_{m} x_{m}
$$


Similarly, condition (3) ensures that

$$
\left(T-\mu_{n}\right) v_{j}=\sum_{m=n+1}^{\infty} \alpha_{m} u_{m, n, j-1}=v_{j-1}
$$

for all $j \in \mathbb{N}$ with $j \geq 2$. Again by the characterization of the algebraic core from Lemma 1, we conclude that

$$
\sum_{m=n+1}^{\infty} \alpha_{m} x_{m} \in C\left(T-\mu_{n}\right) .
$$

On the other hand, by condition (4), we have $x+\sum_{m=1}^{n} \alpha_{m} x_{m} \notin C\left(T-\mu_{n}\right)$ and therefore $y \notin C\left(T-\mu_{n}\right)$. It follows that $\mu_{n} \in \sigma_{T}(y)$ for each $n \in \mathbb{N}$ and hence $\sigma_{T}(y)=\bar{U}$, as desired.

In the preceding result, it is essential to have some condition on the localizable spectrum, not just the spectrum. For instance, if $T$ denotes the unilateral right shift on the Hilbert space $X:=\ell^{2}(\mathbb{N})$, then $T$ is subnormal, $\sigma(T)$ is the closed unit disc, and, by Example 2 of [2], all non-trivial local resolvent functions for $T$ are unbounded. Here $E_{T}(\emptyset)=\{0\}$, because $\bigcap_{n=1}^{\infty} T^{n} X=\{0\}$, while $\sigma_{T}(x)=\sigma(T)$ for all non-zero $x \in X$, so that $\sigma_{\text {loc }}(T)=\emptyset$.

It is an interesting open problem if Theorem 4 remains valid when the requirement that $E_{T}(\emptyset)$ be trivial is weakened to the condition of (SVEP). Of course, it is tempting to apply the method of the preceding proof to the analytic instead of the algebraic core, but substantial difficulties arise at the last step of the proof when trying to establish that $y \notin K\left(T-\mu_{n}\right)$ for all $n \in \mathbb{N}$. This would follow if it could be shown that $\sum_{m=n+1}^{\infty} \alpha_{m} x_{m}$ belonged to $K\left(T-\mu_{n}\right)$, not just to $C\left(T-\mu_{n}\right)$. Theorem 4 is in the same spirit as Theorem 4 of [3], but here the interior of the point spectrum need not be void.

We mention that the bounded local resolvent function constructed in Example 1.2.13 of [10] does not admit a continuous extension to the closure of its natural domain. On the other hand, there exists an example of an operator that admits an everywhere defined non-trivial local resolvent function with continuous derivatives of all orders on $\mathbb{C}$; see $[12]$.

If $T \in L(X)$ and $S \in L(Y)$ are Banach space operators that are intertwined by some injective bounded linear mapping $A: Y \rightarrow X$, in the sense that $T A=A S$, then it is easily seen that (SVEP) for $T$ implies (SVEP) for $S$, that $\sigma_{\text {loc }}(S) \subseteq$ $\sigma_{\text {loc }}(T)$, and that $E_{T}(\emptyset)=\{0\}$ implies that $E_{S}(\emptyset)=\{0\}$. In particular, it follows that the two conditions of Theorem 4 are preserved under quasi-similarity.

As shown in Corollary 6 of [3], given a Banach space $X$ and an arbitrary operator $T \in L(X)$ with (SVEP), the set of vectors $x \in X$ for which the local resolvent function is bounded on $\rho_{T}(x)$ is of the first category in $X$. Nevertheless, this set may be quite large, as illustrated by the following consequences of the preceding result.

Corollary 5. Suppose that $T \in L(X)$ is an operator for which $E_{T}(\emptyset)=\{0\}$ and $\sigma_{\text {loc }}(T)$ has non-empty interior. Then there exists an infinite-dimensional linear subspace $Y$ of $X$ such that, for each $y \in Y$, the local resolvent function $R_{T}(\cdot, y)$ has a continuous extension to $\overline{\rho_{T}(y)}$. In particular, $T$ has a bounded local resolvent function at each $y \in Y$. 
Proof. Since the interior of $\sigma_{\text {loc }}(T)$ is non-empty, we may choose a sequence $\left(U_{n}\right)_{n \in \mathbb{N}}$ of non-empty open discs $U_{n} \subseteq \sigma_{\text {loc }}(T)$ with mutually disjoint closures. For each $n \in \mathbb{N}$, we then apply Theorem 4 with the choice $x:=0$ to obtain some non-zero vector $y_{n} \in X$ with $\sigma_{T}\left(y_{n}\right)=\bar{U}_{n}$ such that $R_{T}\left(\cdot, y_{n}\right)$ has a continuous extension to $\mathbb{C} \backslash U_{n}=\overline{\rho_{T}\left(y_{n}\right)}$.

Since $\sigma_{T}(u+v) \subseteq \sigma_{T}(u) \cup \sigma_{T}(v)$ for all $u, v \in X$, the sequence $\left(y_{n}\right)_{n \in \mathbb{N}}$ is linearly independent. Indeed, given an arbitrary $m \in \mathbb{N}$ and $\alpha_{1}, \ldots, \alpha_{m} \in \mathbb{C}$ for which $\alpha_{1} y_{1}+\cdots+\alpha_{m} y_{m}=0$, we obtain from $-\alpha_{1} y_{1}=\alpha_{2} y_{2}+\cdots+\alpha_{m} y_{m}$ that

$$
\sigma_{T}\left(\alpha_{1} y_{1}\right) \subseteq \sigma_{T}\left(\alpha_{1} y_{1}\right) \cap\left(\sigma_{T}\left(\alpha_{2} y_{2}\right) \cup \cdots \cup \sigma_{T}\left(\alpha_{m} y_{m}\right)\right)
$$

hence $\sigma_{T}\left(\alpha_{1} y_{1}\right) \subseteq \bar{U}_{1} \cap\left(\bar{U}_{2} \cup \cdots \cup \bar{U}_{m}\right)=\emptyset$, therefore, by (SVEP), $\alpha_{1}=0$, and, similarly, $\alpha_{2}=\cdots=\alpha_{m}=0$. Consequently, the linear span $Y$ of $\left(y_{n}\right)_{n \in \mathbb{N}}$ is of infinite dimension.

Finally, given an arbitrary non-zero $y \in Y$, we obtain, after renumbering the vectors $y_{n}$ if necessary, a representation of the form $y=\alpha_{1} y_{1}+\cdots+\alpha_{m} y_{m}$ for suitable $m \in \mathbb{N}$ and non-zero $\alpha_{1}, \ldots, \alpha_{m} \in \mathbb{C}$. Because $\sigma_{T}\left(y_{i}\right) \cap \sigma_{T}\left(y_{j}\right)=\bar{U}_{i} \cap$ $\bar{U}_{j}=\emptyset$ whenever $i \neq j$, we have $\rho_{T}(y)=\rho_{T}\left(y_{1}\right) \cap \cdots \cap \rho_{T}\left(y_{m}\right)$ and $R_{T}(\lambda, y)=$ $\alpha_{1} R_{T}\left(\lambda, y_{1}\right)+\cdots+\alpha_{m} R\left(\lambda, y_{m}\right)$ for all $\lambda \in \rho_{T}(y)$. Thus $R_{T}(\cdot, y)$ admits a continuous extension to $\overline{\rho_{T}(y)}$, as desired.

Corollary 6. Suppose that $T \in L(X)$ satisfies $E_{T}(\emptyset)=\{0\}$ and that $T$ is the quotient of a decomposable operator by a closed invariant subspace. If $X_{T^{*}}^{*}(\partial \sigma(T))=$ $\{0\}$, then the set of vectors $y \in X$ for which the local resolvent function $R_{T}(\cdot, y)$ admits a continuous extension to $\overline{\rho_{T}(y)}$ is dense in $X$. The converse holds provided that $T$ is a normal operator on a Hilbert space.

Proof. We may, of course, assume that $X \neq\{0\}$. Then the condition that $X_{T^{*}}^{*}(\partial \sigma(T))$ be trivial ensures that the interior int $\sigma(T)$ of $\sigma(T)$ is non-empty. Also, by Proposition 2.5.14 of [10], the annihilator of $X_{T}(\operatorname{int} \sigma(T))$ in the dual space $X^{*}$ coincides with $X_{T^{*}}^{*}(\mathbb{C} \backslash \operatorname{int} \sigma(T))=X_{T^{*}}^{*}(\partial \sigma(T))=\{0\}$. Hence, by the Hahn-Banach theorem, $X_{T}($ int $\sigma(T))$ is dense in $X$. On the other hand, as noted above, $\sigma_{\text {loc }}(T)=\sigma(T)$, because $T$ is the quotient of a decomposable operator; see [3] or [11]. Now the first assertion is immediate from Theorem 4.

The final claim follows from Theorem 8 of [3] and the fact that, for a normal operator $T$ with spectral measure $\mathfrak{E}$, the space $X_{T^{*}}^{*}(\partial \sigma(T))$ may be canonically identified with the range of $\mathfrak{E}(\partial \sigma(T))$. Indeed, let $J: X \rightarrow X^{*}$ denote the conjugate-linear isometry from the Hilbert space $X$ onto $X^{*}$ provided by the Riesz representation theorem, and, for an arbitrary closed subset $F$ of $\mathbb{C}$, let $F^{\sharp}:=\{\bar{\lambda}: \lambda \in F\}$. Then the spectral measure $\mathfrak{E}^{\sharp}$ of the Hilbert space adjoint $T^{\sharp} \in L(X)$ satisfies $\mathfrak{E}^{\sharp}\left(F^{\sharp}\right)=\mathfrak{E}(F)$ and therefore $X_{T^{*}}^{*}(F)=J X_{T^{\sharp}}\left(F^{\sharp}\right)=J \operatorname{ran} \mathfrak{E}^{\sharp}\left(F^{\sharp}\right)=J \operatorname{ran} \mathfrak{E}(F)$, by Corollary 1.2 .25 of [10].

\section{Multipliers And CONVOLUtion OPERATORS}

Let $A$ be a semi-simple commutative complex Banach algebra, and let $\Delta(A)$ denote the spectrum of $A$, i.e., the set of all non-trivial multiplicative linear functionals on $A$, endowed, as usual, with the Gelfand topology. Recall from [8] that a mapping $T: A \rightarrow A$ is said to be a multiplier on $A$ provided that $(T u) v=u T v$ for 
all $u, v \in A$. It is well known that each multiplier on $A$ is a continuous linear operator and that the set $M(A)$ of all multipliers on $A$ is a unital closed commutative subalgebra of $L(A)$; see Proposition 4.1 .1 of [10].

Since all multiplication operators are multipliers, $A$ is continuously embedded in $M(A)$, and, in this sense, $M(A)$ contains $A$ as an ideal. Moreover, $M(A)$ inherits semi-simplicity from $A$, and $\Delta(A)$ may be viewed as a subset of the spectrum $\Delta(M(A))$ of the multiplier algebra $M(A)$. For each $T \in M(A)$, there exists a unique continuous bounded complex-valued function $g$ on $\Delta(A)$ for which $\widehat{T u}=g \widehat{u}$ for all $u \in A$, where $\widehat{u}(\varphi):=\varphi(u)$ for all $\varphi \in \Delta(A)$. In fact, $g=\widehat{T} \mid \Delta(A)$, where $\widehat{T}$ is the Gelfand transform of $T$ on $\Delta(M(A))$. Details for all this may be found in [8] or Section 4.3 of [10].

It is also known that the spectrum $\sigma(T)$ of a multiplier $T \in M(A)$ as an operator on $A$ coincides with the spectrum of $T$ as an element of the commutative Banach algebra $M(A)$. Thus, by elementary Gelfand theory, $\sigma(T)=\widehat{T}(\Delta(M(A)))$. The multiplier $T$ is said to have natural spectrum provided that $\widehat{T}(\Delta(A))$ is dense in $\sigma(T)$. This notion originates from the Wiener-Pitt phenomenon in harmonic analysis and is discussed in Sections 4.6 and 4.11 of [10]. In the classical case of group algebras, we will see below that many, but not all, multipliers have natural spectrum.

Theorem 7. Let $A$ be a regular semi-simple commutative Banach algebra, and suppose that $T \in M(A)$ is a multiplier with natural spectrum. Then there exists a non-trivial bounded local resolvent function for $T$ precisely when $\sigma(T)$ has nonempty interior. Moreover, in this case, there exists an infinite-dimensional linear subspace $Y$ of $A$ such that, for each $y \in Y$, the local resolvent function $R_{T}(\cdot, y)$ has a continuous extension to $\overline{\rho_{T}(y)}$.

Proof. First observe that every multiplier $T \in M(A)$ satisfies $\bigcap_{\lambda \in \mathbb{C}}(T-\lambda) A=$ $\{0\}$. Indeed, given $u \in \bigcap_{\lambda \in \mathbb{C}}(T-\lambda) A$ and $\varphi \in M(A)$, let $\lambda:=\widehat{T}(\varphi)$ and choose an element $v \in A$ for which $u=(T-\lambda) v$. Then $\varphi(u)=(\widehat{T}(\varphi)-\lambda) \varphi(v)=0$ and hence, by semi-simplicity, $u=0$. In particular, it follows that $E_{T}(\emptyset)=\{0\}$.

We next claim that $\widehat{T}(\Delta(A)) \subseteq \sigma_{\text {loc }}(T)$ for every $T \in M(A)$. To establish this inclusion, let $\lambda \in \widehat{T}(\Delta(A))$, choose $\varphi \in \Delta(A)$ such that $\lambda=\widehat{T}(\varphi)$, and consider an arbitrary open neighborhood $V$ of $\lambda$. Since $\widehat{T}^{-1}(V) \cap \Delta(A)$ is an open neighborhood of $\varphi$ in the locally compact space $\Delta(A)$, there exist an open set $U$ and a compact set $K$ such that $\varphi \in U \subseteq K \subseteq \widehat{T}^{-1}(V) \cap \Delta(A)$. By the regularity of $A$, we then find some $x \in A$ for which $\widehat{x}(\varphi)=1$ and $\widehat{x} \equiv 0$ on $\Delta(A) \backslash U$. Thus $x \neq 0$ and supp $\widehat{x} \subseteq K$. Since $\widehat{x}$ has compact support, Proposition 4.7 .8 of [10] ensures that $\sigma_{T}(x)=\widehat{T}(\operatorname{supp} \widehat{x}) \subseteq V$ and therefore $0 \neq x \in A_{T}(V)$. Thus $\lambda \in \sigma_{\text {loc }}(T)$, as desired.

In particular, we obtain the identity $\sigma_{\text {loc }}(T)=\sigma(T)$ for every multiplier $T \in$ $M(A)$ with natural spectrum. The assertion is now immediate from Corollary 5 and Proposition 4.7.2 of [10].

Corollary 8. Let $G$ be a locally compact abelian group with dual group $\Gamma$, and suppose that $\mu \in M(G)$ is a regular complex Borel measure on $G$ with natural spectrum, in the sense that $\widehat{\mu}(\Gamma)$ is dense in $\sigma(\mu)$, where $\widehat{\mu}$ denotes the FourierStieltjes transform of the measure $\mu$. Then the operator $T$ of convolution by $\mu$ on the group algebra $L^{1}(G)$ admits a non-trivial bounded local resolvent function if 
and only if $\sigma(\mu)$ has non-empty interior. Moreover, in this case, there exists an infinite-dimensional linear subspace $Y$ of $L^{1}(G)$ such that, for each $y \in Y$, the local resolvent function $R_{T}(\cdot, y)$ has a continuous extension to $\overline{\rho_{T}(y)}$.

The result is immediate from Theorem 7 , since $A:=L^{1}(G)$ is a regular semisimple Banach algebra for which $M(A)$ may be canonically identified with $M(G)$ via convolution; see [8] and [10].

Note that the Wiener-Pitt phenomenon asserts that each non-discrete locally compact abelian group supports a measure without natural spectrum; see Corollary 8.2.6 of [6]. We do not know if the preceding result remains valid for such measures.

In the case of compact groups, a certain special case of the first assertion of Corollary 8 was established in Theorem 16 of [14], based on a completely different approach. By Theorem 4.11.1 of [10], Corollary 8 applies to all measures $\mu \in M(G)$ for which the corresponding convolution operator $T_{\mu}$ is decomposable on $L^{1}(G)$ and hence, in particular, to all measures for which the continuous part is absolutely continuous. It should be noted, however, that, by Theorem 4.11 .10 of [10], for every non-discrete locally compact abelian group $G$, there exists a measure $\mu \in M(G)$ that has natural spectrum while $T_{\mu}$ fails to be decomposable on $L^{1}(G)$.

\section{REFERENCES}

[1] P. Aiena, Fredholm and Local Spectral Theory, with Applications to Multipliers. Dordrecht 2004.

[2] T. Bermúdez and M. GonzÁlez, On the boundedness of the local resolvent function. Int. Eq. Operator Theory 34, 1-8 (1999).

[3] J. BraČIČ ANd V. Müller, On bounded local resolvents. Int. Eq. Operator Theory 55, $477-486$ (2006).

[4] J. Eschmeier and B. Prunaru, Invariant subspaces and localizable spectrum. Int. Eq. Operator Theory 42, 461-471 (2002)

[5] M. González, An example of a bounded local resolvent. Proceedings of the 16 th Conference on Operator Theory in Timişoara, 159-162. Bucharest 1997.

[6] C. C. Graham and O. C. McGehee, Essays in Commutative Harmonic Analysis. New York 1979.

[7] B. E. Johnson and A. M. Sinclair, Continuity of linear operators commuting with continuous linear operators II. Trans. Amer. Math. Soc. 146, 533-540 (1969).

[8] R. Larsen, An Introduction to the Theory of Multipliers. New York 1971.

[9] K. B. Laursen, Algebraic spectral subspaces and automatic continuity. Czech. Math. J. 38 (113), 157-172 (1988).

[10] K. B. Laursen and M. M. Neumann, An Introduction to Local Spectral Theory. Oxford 2000.

[11] T. L. Miller, V. G. Miller, and M. M. Neumann, On operators with closed analytic core. Rend. Circ. Mat. Palermo (2) 51, 495-502 (2002).

[12] V. MüLleR, On smooth local resolvents. Int. Eq. Operator Theory 57, 229-234 (2007).

[13] M. M. Neumann, On local spectral properties of operators on Banach spaces. Rend. Circ. Mat. Palermo (2) Suppl. 56, 15-25 (1998).

[14] M. M. Neumann, Recent developments in local spectral theory. Rend. Circ. Mat. Palermo (2) Suppl. 68, 111-131 (2002).

[15] P. SAPHAR, Contribution à l'étude des applications linéaires dans un espace de Banach. Bull. Soc. Math. France 92, 363-384 (1964). 
V. Müller

Mathematical Institute

Czech Academy of Sciences

Žitná 25

11567 Prague 1

Czech Republic

muller@math.cas.cz
M. M. Neumann

Dept. of Mathematics and Statistics Mississippi State University P.O. Drawer MA

Mississippi State, MS 39762

USA

neumann@math.msstate.edu 